\title{
X-Ray Exposure Time
}

National Cancer Institute

\section{Source}

National Cancer Institute. X-Ray Exposure Time. NCI Thesaurus. Code C69234.

The cumulative time a subject was exposed to X-rays. 